\title{
Was ist Hygiene?
}

\author{
Roland Schulze-Röbbecke
}

Herkunft des Wortes „Hygiene“. In Fach- und Wörterbüchern wird das Wort „Hygiene“ immer wieder von der griechischen Göttin der Gesundheit namens Hygieia (griechisch Y $\gamma \varepsilon i_{\alpha} \alpha$ ) abgeleitet. Schon hier beginnt der Schwindel mit der Hygiene. Das Wort „Hy-

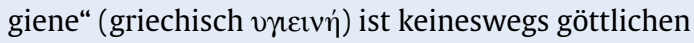
Ursprungs sondern leitet sich von „hygieia“ (vүría), dem griechischen Wort für „Gesundheit“ ab [1] und kann als „Gesundheitslehre“ oder „Gesundheitspflege“ übersetzt werden. Die alten Griechen liebten es, Begriffe wie „Ozean“, „Liebe“ und „Gesundheit“ durch Gottheiten zu personifizieren und diesen dann den entsprechenden Namen zu geben - in den genannten Beispielen also „Okeanos“, „Eros“ und „Hygieia“. Besagte Göttin Hygieia war also nicht namensgebend für die Hygiene sondern führt ihren Namen auf dasselbe Ursprungswort zurück wie das Wort „Hygiene“.

Hygiene im allgemeinen Sprachgebrauch. Wegen dieses etymologischen Schwindels ist die Göttin Hygieia heute dazu verurteilt, bestimmte Hygiene-Gesellschaften, Hygiene-Firmen und Hygiene-Artikel mit ihrem Abbild zu schmücken. Diese haben es offenbar nötig, denn im allgemeinen Sprachgebrauch haftet dem Begriff „Hygiene“ heute nichts Erhabenes mehr an. Er hat vielmehr einen unappetitlichen, gelegentlich sogar obszönen Beigeschmack: Beate Uhse eröffnete 1962 in Flensburg ihren ersten Erotikshop unter dem Namen „Institut für Ehehygiene“. In den SupermarktAbteilungen für „Hygieneartikel“ findet man Deostifte, Papiertaschentücher, Mittel gegen Mundgeruch, Slipeinlagen, Toilettenpapier und Kondome. Wieder wird hier mit der Hygiene geschwindelt: Viele dieser Artikel mögen ihre (ästhetische) Daseinsberechtigung haben; von wirklich gesundheitlicher Relevanz sind sie aber nur zum Teil. Verständlich ist das Bedürfnis, die Peinlichkeit des Umgangs mit „unreinen“ Körperausscheidungen und -gerüchen hinter einem Euphemismus wie „Hygiene“ zu verbergen. Damit hat sich die „Hygiene" in ihrer heutigen umgangssprachlichen Bedeutung aber von der mythologischen und wissenschaftlichen Auffassung von „Gesundheit“ weit entfernt. Im allgemeinen Sprachgebrauch bezeichnet „Hygiene“ heute (eine z.T. irrationale und zwanghafte Auffassung von) Sauberkeit und Keimfreiheit sowie die Abwesenheit oder Beseitigung unreiner, ekelerregender, (vermeintlich) gesundheitsschädlicher Substanzen, Körperausscheidungen und -gerüche, $z$. B. durch Reinigungs- und Desinfektionsmaßnahmen.

Hygiene als medizinisches Fachgebiet. Womit aber befasst sich das an deutschen Universitäten mit einer (rückläufigen) Anzahl von Instituten und Lehrstühlen vertretene medizinische Fach „Hygiene“? Gemäß einer seit über 100 Jahren in Hygiene-Lehrbüchern erscheinenden Definition versucht die Hygiene „Krankheiten zu verhüten sowie das Wohlbefinden und die Leistungsfähigkeit der Menschen zu erhalten bzw. zu steigern“ [2]. Mit diesem universalen Anspruch, der weit über die Ziele der gesamten Präventivmedizin hinaus geht (und z. B. auch das Doping von Radsportlern beinhaltet), hat sich die „Hygiene“ als medizinisches Fachgebiet in unheilvoller Weise übernommen und schuldig gemacht. Angeheizt durch die spektakulären Erfolge bei der Seuchenbekämpfung entstanden aus dem Modefach Hygiene seit Ende des 19. Jahrhunderts Spezialitäten wie Arbeits-, Boden-, Individual-, Kleidungs-, Luft-, Nerven-, Rassen-, Schul-, Seuchen-, Siedlungs-, Sozial-, Wasser- und Wohnungshygiene. Diese wurden während des Nationalsozialismus zu zwei Teilfächern zusammengefasst, der „endogenen Hygiene (Rassenhygiene)“ und der „exogenen Hygiene (Umwelthygiene)“ [3]. Die durch und durch biologistisch und pseudowissenschaftlich geprägte Rassenhygiene kulminierte bekanntlich in den Tötungsfabriken der Nationalsozialisten mit ihren mörderischen SSHygiene-Instituten und ihren monströsen Karrierewissenschaftlern wie Dr. Dr. med. Josef Mengele und Prof. Dr. med. Carl Clauberg. Hier wurde nicht mehr mit der Hygiene geschwindelt; hier wurden im Namen der Hygiene Verbrechen unbeschreiblichen Ausmaßes begangen. - Nach dem zweiten Weltkrieg wurden die Sündenfälle der „Hygiene“ nur zögerlich und halbherzig aufgearbeitet; man tat lieber so, als habe es die Rassenhygiene nie gegeben. Das ohne Rassenhygiene verbleibende Restfach „Hygiene“ befasst sich seit 1945 
nur noch mit der Prävention exogener (d. h. durch Faktoren außerhalb des Körpers verursachter) Gesundheitsstörungen sowie mit der Steigerung von Wohlbefinden und Leistungsfähigkeit [4]. Auf diesem Selbstverständnis beruht auch die heutige Ausbildung zum „Arzt für Hygiene und Umweltmedizin“. Die wissenschaftlichen Aktivitäten des heutigen Faches Hygiene sparen den zweiten Teil seines Aufgabenbereichs („Steigerung von Wohlbefinden und Leistungsfähigkeit“) allerdings weitgehend aus. Erwähnenswerte Teilgebiete des Faches sind heute die Krankenhaus-, Nahrungsmittel-, Trinkwasser- und Umwelthygiene (letztere nicht im nationalsozialistischen sondern in einem neuen Sinne).

Traditionelle deutsche Krankenhaushygiene. Gegen die in Deutschland seit etwa den 1970er Jahren als eigenständiges Teilgebiet der Hygiene praktizierte Krankenhaushygiene ist der Vorwurf erhoben worden, sich hauptsächlich mit baulich-technischen Fragen sowie mit Sauberkeit und Keimfreiheit in der Krankenhausumgebung zu beschäftigen. Tatsächlich hält sich in den Köpfen einiger deutscher Hygieniker, Amtsärzte und anderer Berufsgruppen - besonders aber in den Medien und in der Öffentlichkeit - bis heute die Vorstellung, dass nosokomiale Infektionen (NI) entsprechend der Definition des medizinischen Faches Hygiene exogenen Ursprungs sind, d. h. aus Infektionsquellen außerhalb der infizierten Personen stammen. Gemäß diesem Dogma werden postoperative Wundinfektionen durch Luftkeime und schmutzige OP-Instrumente verursacht, nosokomiale Pneumonien durch verkeimte Beatmungsgeräte und Bakteriämien durch „Krankenhauskeime“ von Oberflächen und Fußböden. Dass die NI-Erreger auch von den betroffenen Patienten selbst stammen können, ist in dieser Vorstellungswelt nicht vorgesehen. Diese traditionelle Auffassung von „Krankenhaushygiene“ lag bis 1997 auch der vom Bundesgesundheitsamt (heute Robert-Koch-Institut) herausgegebenen „Richtlinie für die Erkennung, Verhütung und Bekämpfung von Krankenhausinfektionen“ (heute „Richtlinie für Krankenhaushygiene und Infektionsprävention“) zu Grunde [5]. Die Existenz endogener (aus dem körpereigenen Erregerreservoir verursachter) NI fand erst merkliche Berücksichtigung, als man 1997 begann, die „RKI-Richtlinie“ nach dem heutigen internationalen Wissensstand und den Anforderungen an wissenschaftlich begründete Empfehlungen zu überarbeiten. Dieser stillschweigende Paradigmenwechsel hatte in der „RKI-Richtlinie“ dann auch Stellungnahmen zur Folge, wie z. B. zur Gabe von $\mathrm{H}_{2}$-Blockern und Antazida oder zur Oberkörperhochlagerung im Zusammenhang mit der Prävention endogener no- sokomialer Pneumonien [6]. Endogene NI machen nach heutigem Wissensstand den größten Teil der NI aus. Trotzdem kommen sie in der Wahrnehmung der traditionellen deutschen Krankenhaushygiene, die heute noch immer in einigen Enklaven persistiert, weiterhin nicht vor. Diese anachronistische Einäugigkeit in Verbindung mit Ausdrücken wie „Keimabtötung“, „rein“ und „unrein“ [5,7] zeigt, dass sich die traditionelle deutsche Krankenhaushygiene der ideologischen Altlasten von vor 1945 nicht entledigt hat. Nicht bewusster Schwindel sondern unbewusste ideologische Denkschablonen sind offenbar dafür verantwortlich, dass Sauberkeit und Keimfreiheit der Krankenhausumgebung z.T. heute noch mit der Prävention von NI gleichgesetzt werden. Diese Fehleinschätzung (sieht man vom unbestrittenen Effekt der Händedesinfektion $\mathrm{ab}$ ) hatte z. B. die für Deutschland typische Überbewertung baulicher Gegebenheiten und der routinemäßigen Flächendesinfektion zur Folge, obwohl sich eine hohe Priorität dieser Aspekte mit Hilfe wissenschaftlicher Daten nicht rechtfertigen lässt.

\section{Wissenschaftlich fundierte Krankenhaushygiene.}

Aus den genannten Gründen haben sich die Herausgeber dieser Zeitschrift zunächst schwer getan, das Wort „Krankenhaushygiene“ in den Titel der Zeitschrift aufzunehmen. Schließlich geschah dies aber doch aus Ermangelung an griffigen Alternativen und in der Hoffnung dazu beitragen zu können, den Begriff „Hygiene“ wieder näher an seine ursprüngliche Bedeutung heranzuführen. Allerdings sahen sich die Herausgeber genötigt, den Begriff „Krankenhaushygiene“ im Editorial des ersten Heftes zunächst neu zu definieren als „Prävention von nosokomialen Infektionen und Antibiotikaresistenzen“ unter ausdrücklicher Einbeziehung endogener Infektionen, die völlig andere Präventionsmaßnahmen erfordern als die „Keimabtötung“ in der Umgebung. Diese Definition erschien notwendig, um die Krankenhaushygiene entsprechend heutiger internationaler Gepflogenheiten aus den Grenzen des Faches „Hygiene“ zu lösen, und um sie in einen aktuellen, medizinisch relevanten und der wissenschaftlichen Bearbeitung zugänglichen Kontext zu stellen. Aus wissenschaftlicher Sicht gilt es heute als selbstverständlich, nur solchen krankenhaushygienischen Maßnahmen eine hohe Relevanz einzuräumen, deren präventive Wirksamkeit sich durch geeignete klinische, epidemiologische oder experimentelle Untersuchungen belegen lässt. Dabei werden sowohl exogene als auch endogene NI berücksichtigt. Maßstab für die Wirksamkeit krankenhaushygienischer Maßnahmen sollte die Reduktion der Häufigkeit von NI bzw. der Kolonisation oder Infektion mit multiresistenten Erre- 
gern sein. Der Nachweis von Mikroorganismen in der Krankenhausumgebung ist dagegen als Surrogatmarker meist ohne nennenswerten Aussagewert und nur in bestimmten Fällen (z. B. im Zusammenhang mit Arzneimitteln sowie mit kritischen oder semikritischen Medizinprodukten) überhaupt akzeptabel.

Rechtliche Bedeutung von „Hygiene“ und „Krankenhaushygiene“. Die Begriffe „Hygiene“ und „Krankenhaushygiene" erscheinen in einigen deutscher Rechtsnormen ohne Hinweis darauf, welche der oben erwähnten Bedeutungen gemeint ist. So überträgt $\mathrm{z}$. B. das Gesetz über den öffentlichen Gesundheitsdienst des Landes Nordrhein-Westfalen (NRW) dem öffentlichen Gesundheitsdienst „die Überwachung der Einhaltung der Anforderungen der Hygiene“. Die Krankenhaushygiene-Verordnung NRW verpflichtet dagegen die Träger von Krankenhäusern, „für die Durchführung der notwendigen hygienischen Maßnahmen“ zu sorgen und andere Personen, sich um die „Fortbildung in der Krankenhaushygiene“ zu bemühen. Diese Rechtsnormen verzichten auf jede Definition von „Hygiene“ und „Krankenhaushygiene“, so als ob sie so unmissverständlich wären wie „Vorfahrt“ und „Überholverbot“ in der Straßenverkehrsordnung. Entsprechend verunsichert sind Betroffene und Aufsichtsbehörden bei der Auslegung dieser Rechtsnormen, mit der immer noch anzutreffenden Tendenz, den allgemeinen Sprachgebrauch des Wortes „Hygiene“ oder antiquierte Vorstellungen von „Krankenhaushygiene“ zu Grunde zu legen (s. o.). - Klarere Aussagen über die Bedeutung von „Hygiene“ und „Krankenhaushygiene“ macht das Infektionsschutzgesetz (IfSG). Zwar findet sich in §2 IfSG keine Legaldefinition dieser Begriffe, §23 (2) erwähnt jedoch die „Kommission für Krankenhaushygiene und Infektionsprävention“ beim Robert Koch-Institut (RKI), welche „Empfehlungen zur Prävention nosokomialer Infektionen sowie zu betrieblich-organisatorischen und baulich-funktionellen Maßnahmen der Hygiene in Krankenhäusern und anderen medizinischen Einrichtungen“" erstellt. Der Gesetzgeber lässt somit in §23 (2) IfSG am Beispiel der RKI-Kommission erkennen, was er mit „Krankenhaushygiene“ meint, nämlich die Prävention nosokomialer Infektionen (NI). Dass auch die in §23 (2) erwähnten „betrieblich-organisatorischen und baulich-funktionellen Maßnahmen der Hygiene“ die Prävention von NI bezwecken, lässt der Kommentar [8] vermuten. Eine NI ist wiederum in $\S 2$ definiert als „Infektion mit lokalen oder systemischen Infektionszeichen als Reaktion auf das Vorhandensein von Erregern oder ihrer Toxine, die im zeitlichen Zusammenhang mit einer stationären oder einer ambulanten medizinischen Maßnahme steht, soweit die Infektion nicht bereits vorher bestand“. Wenn sich also Hygiene und Krankenhaushygiene im Sinne des §23 IfSG mit der Prävention von NI befassen, so ist dies nicht auf exogene NI beschränkt. - Weiterhin erscheint im IfSG der Begriff „Hygiene“ in den $\S \S 36,38$ und 41 in den Ausdrücken „Infektionshygiene“, „Hygieneplan“ und Wasserüberwachung ,in hygienischer Hinsicht“. Dass „infektionshygienische Überwachung“ die Überwachung sämtlicher Infektionen meint und dass ein „Hygieneplan“ der Minimierung von Infektionsrisiken dient, geht wiederum aus dem Kommentar hervor [8]. Der Unterschied zwischen den drei im IfSG verwendeten Begriffen „Infektionshygiene“, „Infektionsschutz“, und „Infektionsprävention“ bleibt jedoch unklar.

Missbrauch der „Hygiene“ heute. Durch die fundamental unterschiedlichen Möglichkeiten zur Deutung des Begriffes „Hygiene“ sind Missverständnisse und Missbrauch vorprogrammiert. Dies betrifft z. B. die „Hygieneüberwachung“ medizinischer Einrichtungen durch den öffentlichen Gesundheitsdienst gemäß den gesetzlichen Vorgaben diverser Bundesländer. Einige Gesundheitsämter interpretieren ihre Überwachungspflicht guten Glaubens immer noch in einer Weise, die relevante Aspekte der Infektionsprävention kaum erkennen lassen und eher einem „Sauberkeitsamt“ als einem „Gesundheitsamt“ zur Ehre gereichen. Die Folge ist eine enorme Ressourcenverschwendung und die Unterlassung wirksamer infektionspräventiver Maßnahmen durch falsch gesetzte Prioritäten im Namen der Hygiene. Dieser Missstand hat nichts mit Schwindel zu tun, sondern lässt auf einen bedauernswert schlechten Ausbildungsstand schließen. - In den Bereich des „Schwindels“ fallen dagegen die Aktivitäten mancher Firmen, die darauf abzielen, die in medizinischen Einrichtungen für die „Hygiene“ Verantwortlichen mit ihren Sterilfiltern, Entkeimungsanlagen, antimikrobiellen Wandfarben etc. zu verunsichern. Sofern diese Firmen nicht mit wissenschaftlichen Daten belegen können, dass ihre Produkte nicht nur Keimzahlen reduzieren, sondern auch Infektionen verhindern, liegt der Verdacht nahe, dass nicht die Göttin Hygieia ihre schützende Hand über sie hält sondern Hermes, Schutzgott der Kaufleute und Diebe. 


\section{Literatur}

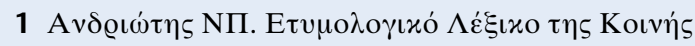

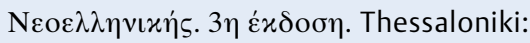

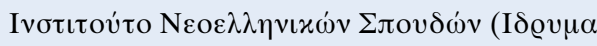

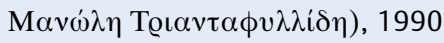

2 Schweinsberg F. Vom Niedergang des Fachs „Hygiene“ in der Medizin. Umweltmed Forsch Prax 2003; 8: 177 - 178

3 Reiter $\mathrm{H}$. Vorschläge zur Neugestaltung des Hygieneunterrichts und zur Sicherung des Nachwuchses auf dem Gebiet der Hygiene. In: Reiter H. Das Reichsgesundheitsamt 1933-1939 - Sechs Jahre nationalsozialistische Führung. Berlin: 1939

4 Pschyrembel - Klinisches Wörterbuch. 259. Aufl. Berlin, New York: Walter de Gruyter, 2002

5 Robert Koch-Institut (Hrsg). Richtlinie für Krankenhaushygiene und Infektionsprävention - Alte Anlagen der Richtlinie für Krankenhaushygiene und In- fektionsprävention. München, Jena: Urban \& Fischer, 2004. www.rki.de > Infektionsschutz > Krankenhaushygiene $>$ Empfehlungen der Kommission für Krankenhaushygiene > Weitere Empfehlungen (vor 1997)

6 Kommission für Krankenhaushygiene und Infektionsprävention am Robert Koch-Institut. Prävention der nosokomialen Pneumonie. Bundesgesundheitsbl Gesundheitsforsch Gesundheitsschutz 2000; 43: $302-309$

7 Kommission für Krankenhaushygiene und Infektionsprävention beim Robert Koch-Institut. Anforderungen an die Hygiene bei der Reinigung und Desinfektion von Flächen. Bundesgesundheitsbl Gesundheitsforsch Gesundheitsschutz 2004; 47: $51-61$

8 Bales S, Baumann HG, Schnitzler N. Infektionsschutzgesetz - Kommentar und Vorschriftensammlung. Stuttgart, Berlin, Köln: Kohlhammer, 2001 